\title{
Criminologie
}

\section{Déjudiciarisation ou non-judiciarisation : variation des tendances entre auteurs présumés - accusés ou traités hors cour}

\section{Sandra Longtin}

Volume 35, numéro 1, printemps 2002

L'âge et la question criminelle

URI : https://id.erudit.org/iderudit/027517ar

DOI : https://doi.org/10.7202/027517ar

Aller au sommaire du numéro

Éditeur(s)

Les Presses de l'Université de Montréal

ISSN

0316-0041 (imprimé)

1492-1367 (numérique)

Découvrir la revue

Citer cet article

Longtin, S. (2002). Déjudiciarisation ou non-judiciarisation : variation des tendances entre auteurs présumés - accusés ou traités hors cour. Criminologie, 35(1), 133-159. https://doi.org/10.7202/027517ar
Résumé de l'article

La présente étude compare Les données de la criminalité au Québec, de 1991 à 1998, chez les jeunes auteurs présumés - accusés ou traités hors cour - âgés de 12 à 17 ans. Sur cette période, nous avons constaté une augmentation constante du taux déjeunes traités hors cour et une baisse soutenue de celui des accusés. Ces variations s'observent à des degrés différents pour les trois grandes catégories d'infractions du Programme de déclaration uniforme de ta criminalité (DUC) : crimes de violence, crimes contre la propriété et autres crimes. L'absolue volonté de déjudiciariser ne suffit pas, à elle seule, à expliquer ces changements. Il y aurait lieu de tenir compte des politiques administratives reposant sur une logique économique. Il faut bien savoir que, sous le couvert du concept de déjudiciarisation, c'est le pouvoir discrétionnaire du poursuivant qui est de plus en plus sollicité. 


\title{
Déjudiciarisation ou non- judiciarisation : variation des tendances entre auteurs présumés - accusés ou traités hors cour
}

\author{
Sandra Longtin \\ Ph.D. Criminologie \\ slongtin@qc.aira.com
}

RÉSUMÉ - La présente étude compare les données de la criminalité au Québec, de 1991 à 1998, chez les jeunes auteurs présumés - accusés ou traités hors cour - àgés de 12 à 17 ans. Sur cette période, nous avons constaté une augmentation constante du taux de jeunes traités hors cour et une baisse soutenue de celui des accusés. Ces variations s'observent à des degrés différents pour les trois grandes catégories d'infractions du Programme de déclaration uniforme de la criminalité (DUC) : crimes de violence, crimes contre la propriété et autres crimes. L'absolue volonté de déjudiciariser ne suffit pas, à elle seule, à expliquer ces changements. Il y aurait lieu de tenir compte des politiques administratives reposant sur une logique économique. Il faut bien savoir que, sous le couvert du concept de déjudiciarisation, c'est le pouvoir discrétionnaire du poursuivant qui est de plus en plus sollicité.

ABSTRACT - The present study compares the data on criminality for young offenders, accused and apprehended, aged 12 to 17 in Quebec, for 1991 to 1998. During this period, the rate of offenders apprehended has increased constantly while the rate of offenders being accused has decreased. These changes can be observed at various degrees for the three categories of offences considered in the Uniform Crime Report (UCR) : violence, property and others. It is suggested that the absolute desire of diversion is not sufficient to explain by itself the change. Administrative guidelines based on an economic logic may be taken into account. Under the concept of diversion it is the discretionary power vested in the prosecution that is more and more solicited. 


\section{Introduction}

Carrington (1999) a mené, à l'aide des données du Programme de déclaration uniforme de la criminalité (DUC), une analyse sur l'évolution de la criminalité des jeunes au Canada de 1977 à 1996. Il ressort de cette analyse que le Québec se distingue des autres provinces par une diminution de ses jeunes accusés per capita en 1984-1985 par rapport à la période précédente de 1980-1983. De plus, entre 1980 et 1988 , le Québec a affiché le plus haut taux de jeunes appréhendés, mais le plus bas taux de jeunes accusés. Enfin, ce phénomène aura été particulièrement évident en 1996. Ces tendances spécifiquement québécoises, avancent d'aucuns, pourraient être attribuables au système de déjudiciarisation introduit en 1979 par la Loi sur la protection de la jeunesse, loi sur l'application de laquelle n'aurait pas eu d'effet le remplacement de la Loi sur les jeunes délinquants par la Loi sur les jeunes contrevenants en 1984 (Le Blanc et Beaumont, 1988 ; 1992). Malgré les changements apportés par la loi de 1984 à la façon de traiter les jeunes contrevenants, nous observons, surtout pour les années 90 , d'importantes fluctuations des changements constatés dans le traitement des jeunes auteurs présumés, qu'ils soient accusés ou traités hors cour.

Nous pensons que l'absolue volonté de déjudiciariser certains cas ne suffit pas à elle seule à expliquer ces changements. N'y aurait-il pas lieu de tenir compte des politiques administratives fondées sur une logique purement économique qui ont une incidence sur les pratiques québécoises ? Car il faut bien voir que, sous le couvert du concept de déjudiciarisation, c'est le pouvoir du poursuivant qui est de plus en plus sollicité.

La présente étude compare les données de la délinquance chez les jeunes auteurs présumés de 12 à 17 ans pour la période de 1991 à 1998, qui, rappelons-le, n'a pas été une période de transition législative. On ne peut donc attribuer l'évolution du traitement des jeunes à l'introduction d'une nouvelle loi ou à d'importantes modifications d'une loi préexistante susceptibles d'influer de manière significative sur les résultats. Nous examinons premièrement la tendance de la délinquance chez les jeunes et, deuxièmement, ce qui se dégage de la comparaison des données relatives aux jeunes accusés et aux jeunes traités hors cour. 


\section{Méthodologie}

Les données policières sur la criminalité au Québec sont les données du Programme de déclaration uniforme de la criminalité (DUC), telles qu'elles sont extraites et publiées par le ministère de la Sécurité publique du Québec. Pour mémoire, la $D U C$ est une banque pancanadienne où sont stockés des renseignements relatifs aux infractions criminelles qui ont été signalées aux services de police et ont fait l'objet d'enquêtes, que des accusations aient été portées (accusés ${ }^{1}$ ) ou non (traités hors cour ${ }^{2}$ ). Fait à signaler, la $D U C$ sous-estime le nombre de crimes commis. La raison en est qu'elle ne considère que les crimes connus de la police et est orientée vers les crimes les plus graves, donc les plus susceptibles d'être déclarés. La DUC sous-évalue également la criminalité juvénile, les services de police étant moins portés à rapporter les méfaits commis par les jeunes (Doob et Chan, 1982). En revanche, ses données sur les tendances de la criminalité sont fiables.

Nous avons calculé le pourcentage d'auteurs présumés - accusés ou traités hors cour - au Québec pour chaque année de la période étudiée (1991-1998) et nous avons ensuite reporté les résultats de chacune sur ceux de l'année 1991 (voir en annexe : tableaux 1 à 8).

1. Le policier classe une affaire « mise en accusation * lorsqu'au moins une accusation est déposée contre au moins une personne.

2. Le policier peut classer " sans mise en accusation " s'il peut répondre oui à chacune des questions suivantes :

- Le contrevenant a-t-il été identifié ?

- Y a-t-il suffisamment de preuves pour déposer une dénonciation?

- La police exerce-t-elle un pouvoir discrétionnaire ou existe-t-il d'autres taisons indépendantes de sa volonté qui l'empêchent de déposet une dénonciation?

Dans le manuel $D U C$, on indique que les cas où il est possible de classer une infraction " sans mise en accusation » sont limités aux exemples suivants :

1. Le contrevenant est décédé avant que les accusations ne soient portées. Si le contrevenant a été accusé avant de mourir, l'infraction peut être classée par mise en accusation.

2. Le contrevenant est âgé de moins de 12 ans.

3. Une directive a été temise à l'extérieur de votre service, par exemple par le Procureur général ou son représentant, qui vous ordonne de ne pas intenter de poursuites dans certaines circonstances.

4. Le contrevenant a été admis dans un hôpital psychiattique, et il est peu probable qu'il soit remis en liberté.

5. Étant donné les circonstances, la police a exercé un pouvoir discrétionnaire et a décidé de ne pas porter d'accusation, bien que l'accusé [sic] ait été identifié et qu'il y ait suffisamment de preuves pour le faire.

6. Le contrevenant a été identifié, mais il existe un obstacle précis aux procédures, par exemple l'immunité diplomatique. 


\section{Changements dans les pratiques}

L'observation du nombre de jeunes auteurs présumés dans les trois grandes catégories d'infractions au Code criminel (crimes de violence, crimes contre la propriété et « autres crimes") met en évidence un changement substantiel dans le traitement de ces jeunes contrevenants. Au cours de la période à l'étude, le taux d'auteurs présumés traités hors cour augmente de façon soutenue jusqu'à dépasser celui, en constante diminution, des accusés. Ces variations s'observent à des degrés divers dans les trois grandes catégories précitées.

Pour ce qui est des crimes de violence, le taux d'accusés progresse de $10 \%$, contre $149 \%$ pour les jeunes traités hors cour ${ }^{3}$.

Quant à la catégorie des crimes contre la propriété, le taux d'accusés baisse de $52 \%$, alors que le nombre de jeunes traités hors cour accuse une hausse de $107 \%$.

7. Le plaignant ou le témoin principal est décédé, et aucune poursuite ne peut, par conséquent, être engagée.

8. Le contrevenant est connu et des preuves suffisantes ont été réunies contre lui, mais le plaignant refuse de le poursuivre, ou ce dernier connaît le ou les contrevenants mais tefuse de divulguer leut nom à la police; il ne s'agit pas d'une infraction " non fondée $*$.

9. L'accusé purge déjà une peine, et il serait inutile de déposer une dénonciation.

10. Le contrevenant se trouve dans un pays étranger et ne peut être extradé (ou il s'agit d'une infraction à une loi provinciale ou à un règlement municipal et le suspect se trouve dans une autre province).

11. Lorsqu'il s'agit de jeunes contrevenants, l'infraction est classée sans mise en accusation dans les cas suivants : le cas du jeune contrevenant a été soumis à un organisme de sélection chargé de prendre une décision quant à la mise en accusation ; le contrevenant est remis en détention (par exemple dans un centre d'éducation surveillée).

12. Le contrevenant a commis plus d'une inftaction à des moments diffétents, dans des endroits différents et dans des circonstances différentes. Il a été convenu d'accuser le contrevenant de la plus grave de ces infractions, puisqu'il serait inutile d'engager des poursuites relativement aux autres infractions.

Depuis 1996, les corps policiers distinguent, sous $D U C 2$, les cas d'application de mesures de rechange.

3. Cette catégorie, également désignée comme "crimes contre la personne", comprend les homicides, les tentatives de meurtre, les inftactions d'ordre sexuel, les voies de fait, les vols qualifiés et les enlèvements. Les voies de fait représentent $79 \%$, les homicides, $0,2 \%$.

4. On retrouve dans cette catégorie les introductions par effraction, les vols de véhicules moteurs, les vols de plus et de moins de $5000 \$$. Les vols de moins de $5000 \$$ constituent $59 \%$ de cette catégorie. 
Pour la catégorie "autres crimes », le taux d'accusés diminue de $27 \%$, tandis que celui des jeunes traités hors cour augmente de $24 \%{ }^{5}$ (ministère de la Sécurité publique, 1999).

Nous assistons à un changement effectif dans les pratiques, qui ne peut être attribué à la loi ou à un remaniement de celle-ci. À preuve, la loi de 1984 a été modifiée à deux reprises, en 1986 et en 1992 . La seconde réforme a porté à cinq ans les peines applicables en matière d'homicide et modifié les dispositions relatives aux renvois devant les tribunaux pour adultes (Trépanier, 1999).

Nous suggérons donc que les pratiques policières et les politiques administratives ont une incidence sur les taux de mises en accusation. La comparaison des tendances des deux groupes de jeunes auteurs permettra d'en apprécier l'impact.

Les changements observés se situent en amont de l'entrée dans le système judiciaire, au stade de l'intervention entre l'agent de police et le jeune auteur présumé, et à celui des décisions, de nature à influencer le traitement de la criminalité juvénile, que prend le substitut du procureur général. On peut done raisonnablement postuler que les variations dans les taux d'accusation peuvent s'expliquer par les changements apparus dans la façon dont les acteurs du système judiciaire exercent leur pouvoir discrétionnaire.

\section{Le poids de l'économisme}

Sur la scène socio-économique, la récession et l'austérité budgétaire marquent les années 1990, au Québec comme ailleurs. Les statistiques du recensement de 1991 révèlent un accroissement du nombre de familles monoparentales, une concentration des personnes âgées et des célibataites dans les centres urbains et une préférence pour la périphérie des centres chez les familles avec enfants. Il en ressort également que le taux de chômage, lequel demeure élevé (10 \%) pour l'ensemble de la population,

5. Cette catégorie inclut la possession de biens volés, les fraudes (Statistique Canada, contrairement au ministère de la Sécurité publique du Québec, classe la possession de biens volés et la fraude dans la catégorie "crime contre la propriété ", voir Juristat : Statistiques de la criminalité au Canada, 1998 (1999)), la prostitution, les jeux et paris, les armes offensives, les méfaits et les " auttes infractions » au Code criminel. Cette dernière sous-catégorie couvre la monnaie contrefaite, les crimes d'incendie, les menaces, les appels téléphoniques harcelants, ainsi que les défauts de se conformer à une ordonnance de probation ou à un engagement contracté devant un juge. 
atteint $22 \%$ chez les moins de vingt ans. On assiste aussi à une relocalisation industrielle. Cette période se distingue encore par la remise en question du partage des responsabilités entre paliers de gouvernement, mais surtout par la rationalisation des budgets gouvernementaux (Quesnel, 1999).

La volonté inflexible des gouvernements d'atteindre l'équilibre budgétaire au cours de la décennie 1990 semble avoir eu des répercussions jusque dans les pratiques des intervenants du système judiciaire. Au cours de cette période, à infractions identiques, davantage de dossiers de jeunes auteurs présumés sont traités hors cour, si bien qu'un plus grand nombre de ces jeunes bénéficient de mesures de substitution, dites "de rechange ». Or, ni le traitement hors cour ni la mesure de rechange ne donne lieu à un casier judiciaire. La volonté, de plus en plus évidente, de ne pas mettre en branle la totalité du processus judiciaire a pour corollaire le recours systématique au pouvoir discrétionnaire de la poursuite, qui peut avoir des effets positifs s'il est bien structuré, mais qui peut aussi mener à des abus, c'est-à-dire, en clair, à des inégalités de traitement, comme nous le verrons plus loin.

\section{Les tendances de la délinquance chez les jeunes}

La figure 1.1 présente la distribution, par 100000 jeunes au Québec, de l'ensemble des jeunes auteurs présumés pour chacune des trois catégories de la DUC. Une légère diminution s'observe chez l'ensemble des auteurs présumés : 5023 en 1991 contre 4884 en 1998, soit un recul de $3 \%$. La moyenne pour ces huit années s'établit à 4383 auteurs présumés. Un sommet apparait en 1993, soit une augmentation de $22 \%$ par rapport à l'année précédente, suivie d'une baisse de $24 \%$ en 1994 . Une tendance similaire s'observe dans la catégorie des crimes contre la propriété où, pour la même période, se constate une progression de $20 \%$ à laquelle fait suite une régression de $26 \%$. Il en va de même dans la catégorie " autres crimes » où, à une hausse de $34 \%$, succède une baisse de $33 \%$. Le sommet de 1993 ne peut donc être imputé aux auteurs de crimes de violence, dont le nombre est en progression plutôt constante.

La figure 1.2 donne le pourcentage de chacune des catégories. Au fil des ans, le taux d'auteurs présumés de crimes contre la propriété est passé graduellement de 60 à $52 \%$ de l'ensemble des auteurs présumés. En revanche, celui des auteurs présumés de crimes de violence a progressé de 14 à $21 \%$. La proportion des auteurs présumés d' " autres crimes » est demeurée relativement stable, autour de $27 \%$. 
FIGURE 1.1

Auteurs présumés de 12 à 17 ans au Québec par 100000 jeunes de population et par catégorie d'infractions

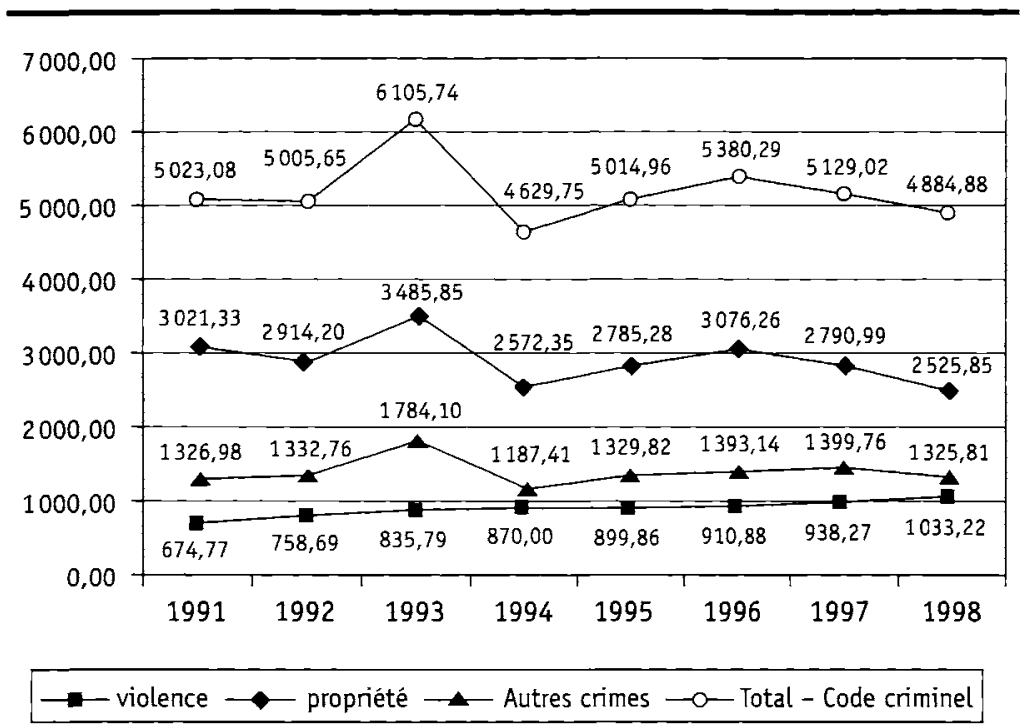

FIGURE 1.2

Pourcentage annuel des auteurs présumés pour les trois catégories d'infraction au Code Criminel

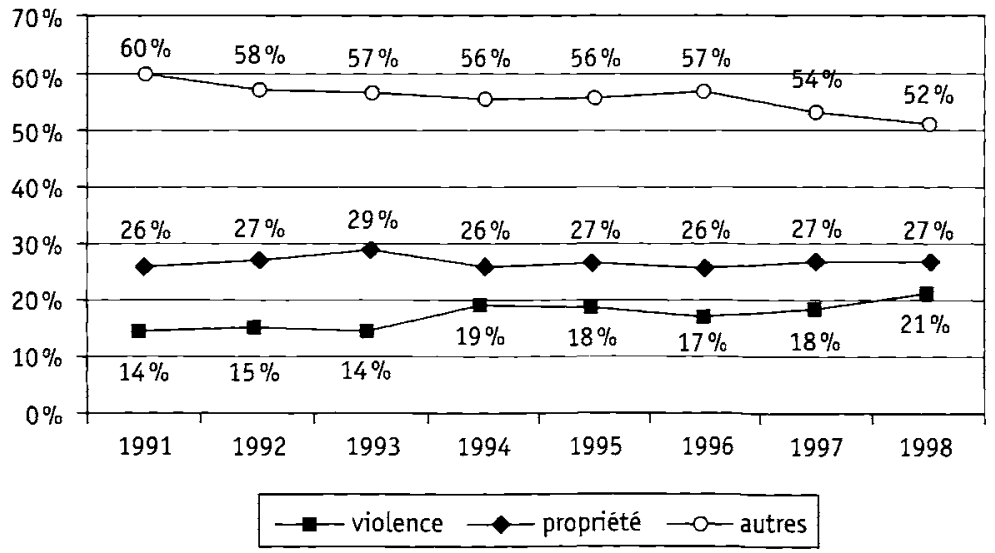




\section{Les auteurs présumés d'infractions au Code criminel}

La figure 2.1 donne une vue d'ensemble des auteurs présumés par 100000 jeunes. Le nombre d'accusés, 3618 en 1991, est passé à 2225 en 1998 (moyenne : 2 904), en baisse de $38 \%$. Le nombre des jeunes traités hors cour, 1404 en 1991, s'élève à 2629 en 1998 (moyenne : 2 242), en hausse de $87 \%$.

Plus révélatrice encore est la figure 2.2 , où nous observons que, malgré les fluctuations d'une année à l'autre du nombre d'auteurs présumés, les pourcentages de jeunes accusés et traités hors cour varient selon une courbe régulière. Pour la période à l'étude, le pourcentage annuel d'accusés passe de 72 à $46 \%$; en revanche, celui des jeunes traités hors cour progresse de 28 à $54 \%$. Depuis 1996, un jeune interpellé a donc plus de chances de bénéficier d'un traitement hors cour.

\section{Infractions au Code criminel}

FIGURE 2.1

Auteurs présumés, accusés et traités hors cour àgés de 12 à 17 ans par 100000 jeunes de population au Québec

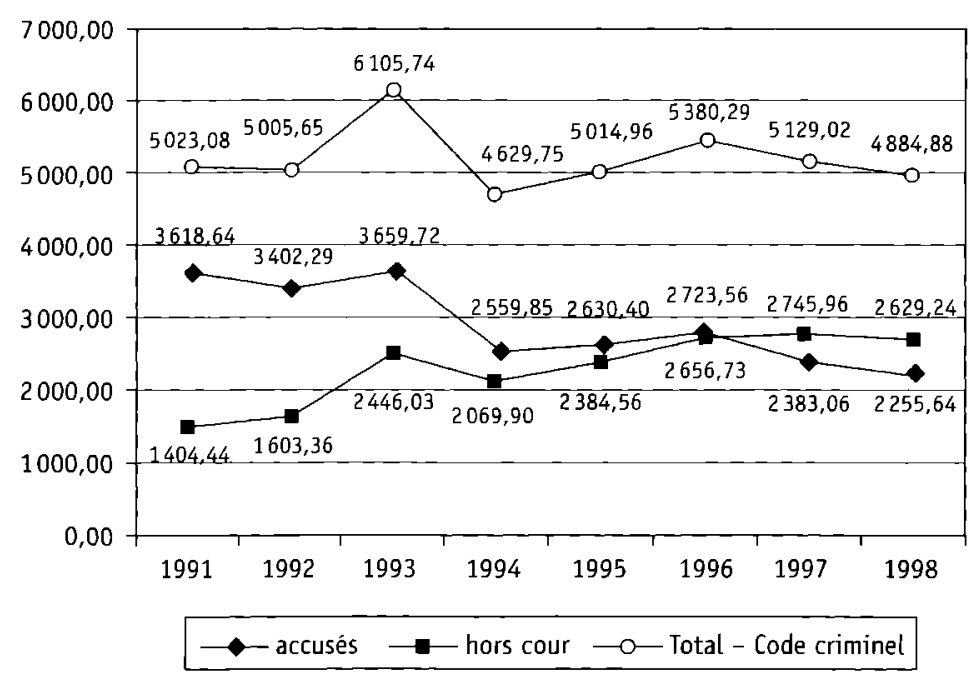


FIGURE 2.2

Les taux d'auteurs présumés accusés ou traités hors cour

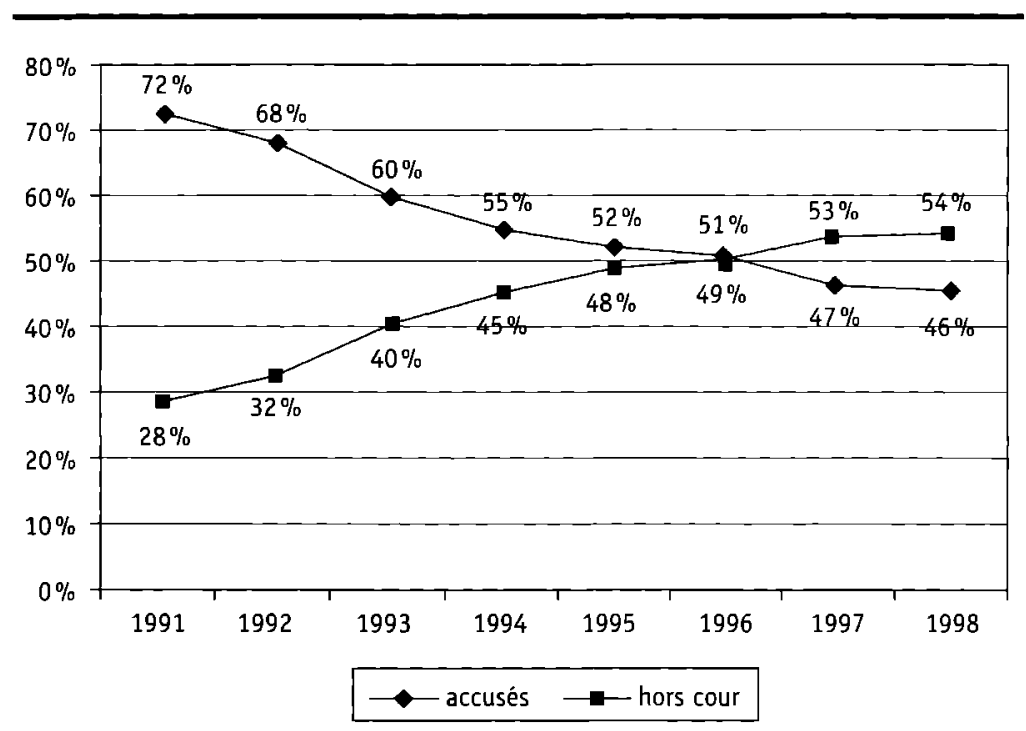

\section{Les crimes de violence}

La figure 3.1 montre l'évolution du nombre d'auteurs présumés de crimes de violence par 100000 jeunes. Ce nombre, de 675 en 1991 , atteint 1033 en 1998, pour une augmentation de $53 \%$. Le nombre d'accusés demeure relativement stable au cours de cette période : 475 en 1991, comparativement à 529 en 1998 (moyenne : 502), soit un accroissement de $11 \%$ seulement. Toutefois, le nombre de cas traités hors cour monte en flèche - 199 en 1991 contre 504 en 1998 (moyenne : 363 ) - pour atteindre $153 \%$. Autrement dit, nonobstant la croissance du nombre des auteurs présumés, ce sont les chiffres relatifs aux traités hors cour qui sont en hausse, alors que ceux des accusés baissent.

La figure 3.2 corrobore ce constat : au cours de cette période, le pourcentage d'accusés passe graduellement de 71 à $51 \%$ et le taux de traités hors cour, de 30 à $49 \%$. 
142 CRIMINOLOGIE, VOL. $35 \mathrm{~N}^{\circ} 1(2002)$

FIGURE 3 . 1

Auteurs présumés, accusés et traités hors cour àgés de 12 à 17 ans par 100000 jeunes de population au Québec

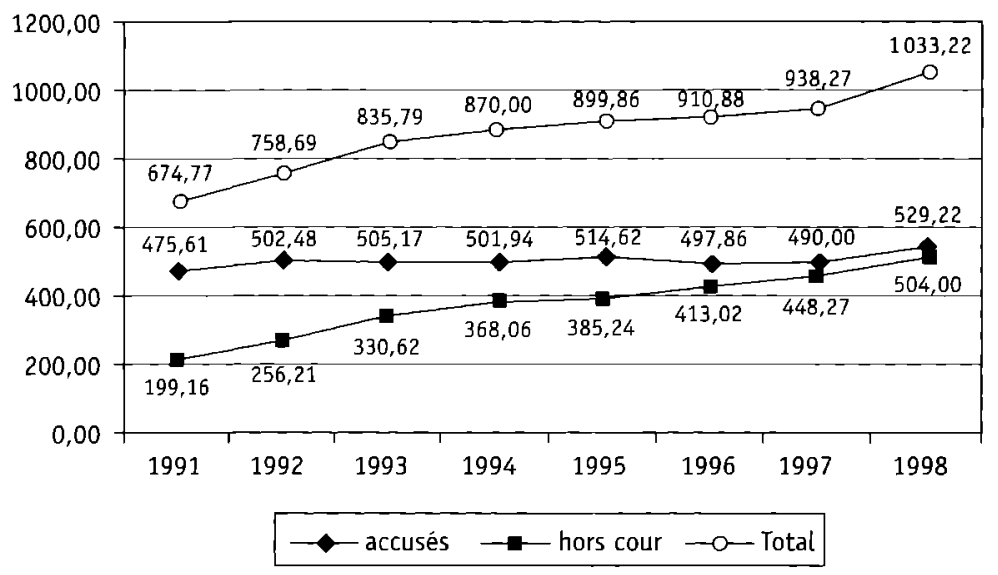

FIGURE 3.2

Les taux d'auteurs présumés accusés ou traités hors cour

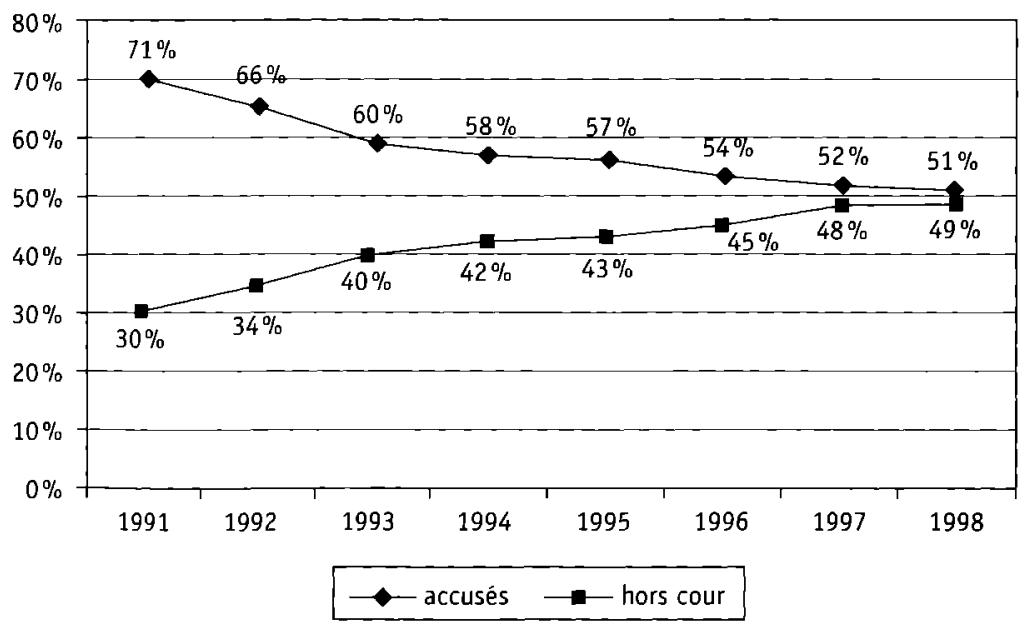




\section{Les crimes contre la propriété}

La figure 4.1 révèle que le nombre d'auteurs présumés de crimes contre la propriété par 100000 jeunes est en baisse, nonobstant quelques variations : 3021 en 1991 contre 2525 en 1998, ou -16\%. Le nombre d'accusés, en net recul, s'établit à 1381 en 1998 comparativement à 2364 en 1991, soit un écart de $52 \%$. Ainsi, au fil des ans, le nombre d'accusés finit par dépasser celui des individus traités hors cours, lequel progresse de 658 en 1991 à 1145 en 1998, faisant un bond de $109 \%$.

La figure 4.2 illustre l'évolution des pourcentages d'accusés et d'individus traités hors cour rapportés aux auteurs présumés de crimes contre la propriété. Celui des accusés, de $78 \%$ en 1991, s'établit à $45 \%$ en 1998. En revanche, celui des individus traités hors cour, de $22 \%$ en 1991, atteint $55 \%$ en 1998 . En comparant les deux figures il ressort que, quel que soit le nombre d'auteurs présumés et sa fluctuation dans le temps, le pourcentage des accusés régresse de façon constante depuis 1996, jusqu'à devenir inférieur à celui des individus traités hors cour.

FIG URE 4 . 1

Auteurs présumés, accusés et traités hors cour àgés de 12 à 17 ans par 100000 jeunes de population au Québec

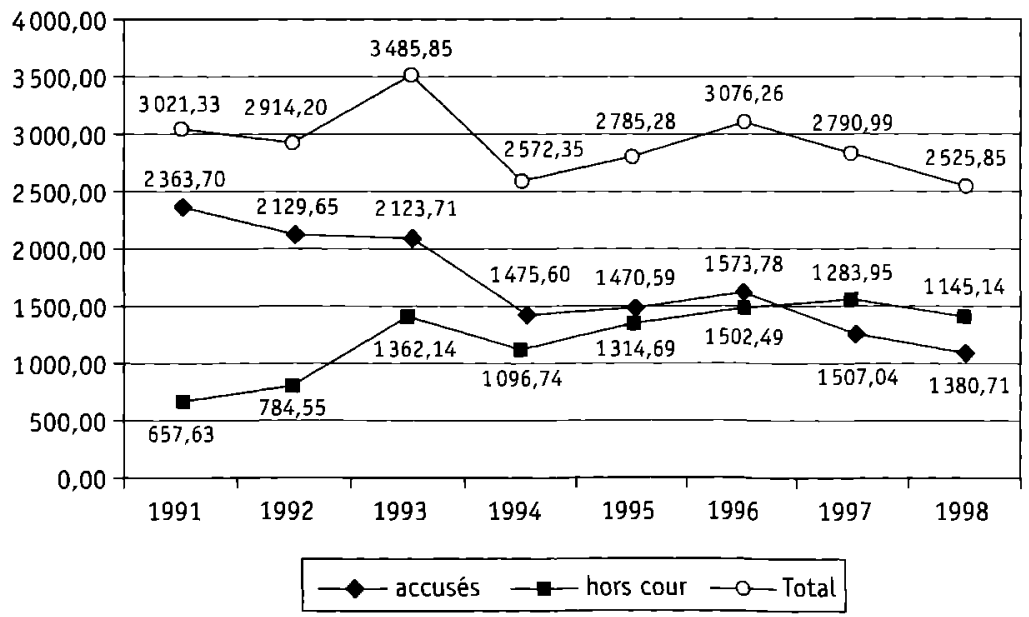


FIGURE 4.2

Les taux d'auteurs présumés accusês ou traités hors cour

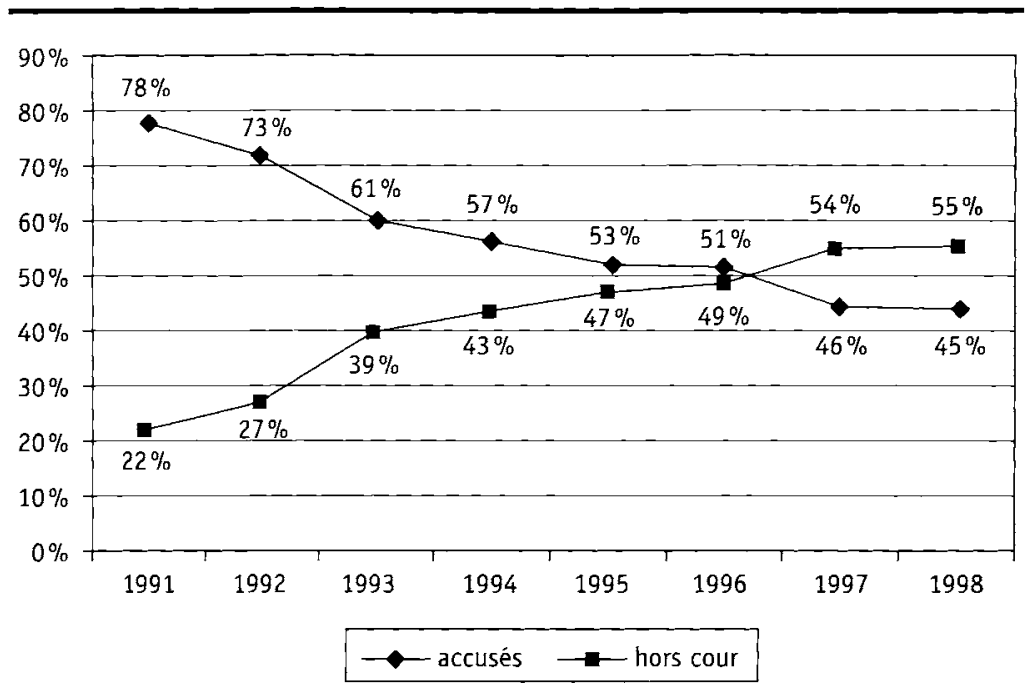

\section{Les auteurs présumés d'autres infractions}

La figure 5.1 montre que, malgré un sommet inusité en 1993, le nombre d'auteurs présumés d' " autres crimes " par 100000 jeunes demeure assez stable au cours des années 1991-1998 (moyenne : 1 385). Pourtant, entre ces deux dates, le nombre d'accusés fléchit : 779 à 581 (moyenne : 706), soit une régression de $25 \%$; et les individus traités hors cour, à l'inverse, passent de 547 à 745 (moyenne: 678), soit une progression de $36 \%$.

Ainsi que le révèle la figure 5.2 , la proportion d'accusés, qui représente $59 \%$ de l'ensemble des jeunes auteurs présumés en 1991, n'est plus que de $44 \%$ en 1998. Au cours de la période concernée, celle des individus traités hors cour croît de 41 à $56 \%$. Donc, pour cette catégorie, le pourcentage des individus traités hors cour dépasse celui des accusés depuis 1994, comparativement à 1996 pour la catégorie des crimes contre la propriété.

Cette analyse permet le constat suivant : depuis 1991, le traitement des jeunes a subi une transformation significative. Pour les deux premières catégories, la proportion des accusés, à l'origine supérieure à $70 \%$, a décrû graduellement pour passer sous la barre des $50 \%$. Dans 
FIGURE 5.1

Auteurs présumés, accusés et traités hors cour âgës de 12 à 17 ans par 100000 jeunes de population au Québec

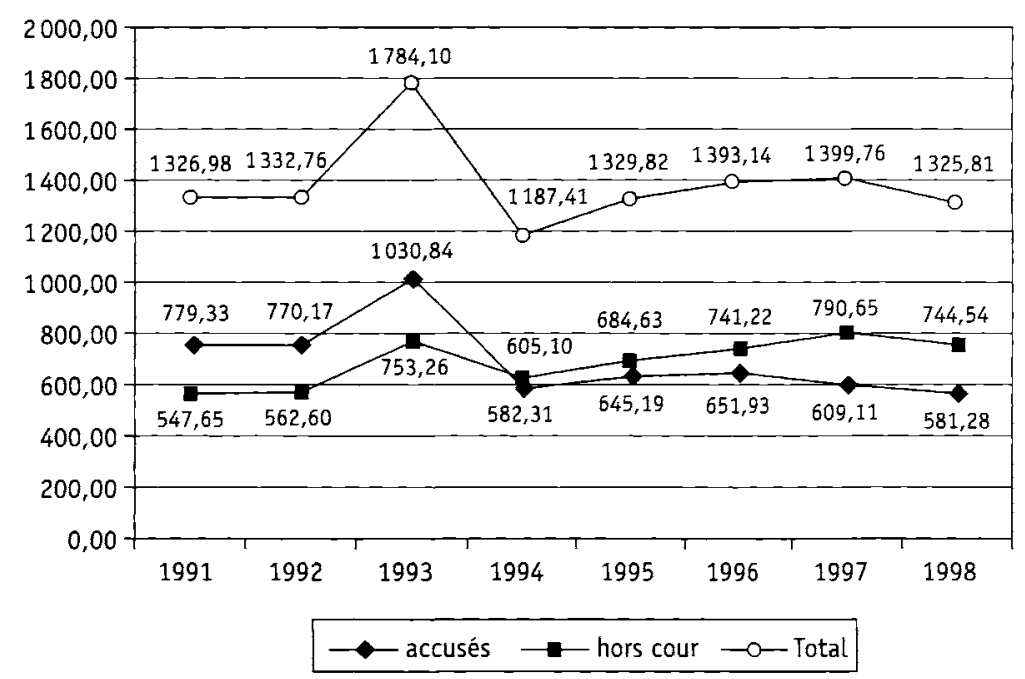

FIGURE 5.2

Les taux d'auteurs présumés accusés ou traités hors cour

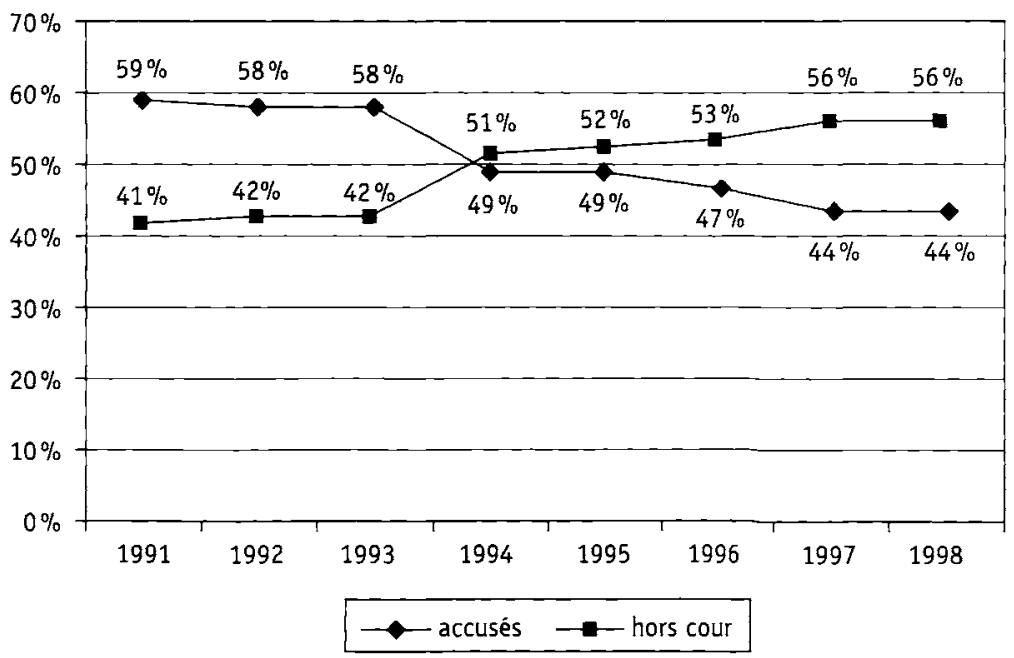


la dernière catégorie (" autres crimes »), la variation est pratiquement aussi importante, le pourcentage des accusés, qui frôlait au départ les $60 \%$, s'établissant à moins de $45 \%$.

Au Québec, il est incontestable que les intervenants auprès des jeunes préconisent les mesures de rechange (Coalition pour la justice des mineurs, 1999). Mais, selon nos résultats, nous ne pouvons écarter l'hypothèse selon laquelle des politiques administratives fondées sur une logique économique et/ou budgétaire favoriseraient le traitement hors cour des jeunes. Dans ce processus d'atténuation de la répression présenté sous le couvert vertueux de la déjudiciarisation, ce serait le pouvoir discrétionnaire du poursuivant qui serait le plus souvent sollicité. Mais qu'en est-il au juste?

\section{Le pouvoir discrétionnaire}

En exerçant leur pouvoir discrétionnaire, les agents de police épargnent aux jeunes auteurs présumés d'infractions au Code criminel des démêlés avec le système judiciaire. Devant une première infraction, relativement mineure par surcroît, l'agent de la paix peut choisir de ne pas judiciariser et opter pour le traitement du cas de façon informelle (avertissement/ rappel de la loi, discussion avec les parents). Il peut également confier à un organisme communautaire le jeune qui y consent (Savoie, 1999).

La discrétion est au cœur du travail policier. Elle s'exerce dès lors que l'agent a toute latitude pour choisit entre plusieurs actions possibles (Davis, 1969). Il est tout simplement irréaliste de penser qu'on peut codifier tous les aspects du travail policier. Il arrive toujours un moment où le justiciable dépend du "bon jugement » de l'agent. En tant que premier intervenant du système judiciaire, la police joue un rôle central en choisissant la façon dont sera traité l'adolescent :

La loi ne écrit pas en détail les situations où les dossiers devraient faire l'objet d'un renvoi ou être fermés ; toutefois, le pouvoir discrétionnaire dont jouit la police permet à celle-ci d'adapter ses interventions et de prendre ses décisions en se fondant sur les circonstances particulières de l'adolescent et sur la prétendue infraction (MacKillop, 1998).

D'autres acteurs du système jouissent d'un pouvoir discrétionnaire. Par exemple, le substitut du procureur général, lorsqu'il décide de procéder ou non dans un cas donné, et le juge aussi, quand il statue sur la recevabilité d'une variété de requêtes, comme l'admissibilité des preuves. Le système judiciaire peut même être vu comme une suite de 
décisions discrétionnaires qui s'enclenche avec la décision d'arrêter un suspect et prend fin avec le sortie de ce dernier du système (Gottfredson et Gottfredson, 1988 ; Gaines et al., 1999).

L'Administration s'efforce d'encadrer la discrétion des agents de police par des politiques et des orientations traduites dans des directives, des ordres et la hiérarchie. Les agents pénaux conservent malgré tout un pouvoir considérable dans la façon d'appliquer la loi, de dispenser des services et d'obéir aux ordres. Ils utilisent leur pouvoir discrétionnaire dans une variété de décisions au jour le jour : appliquer ou non une loi spécifique; mener ou non une enquête ; rechercher ou non un individu ; procéder ou non à une arrestation, une fouille ; interroger ou non un suspect, le détenir ou le déférer au procureur général ; rédiger ou non un rapport officiel; poursuivre ou non l'enquête sur un crime particulier. Les mêmes circonstances suspectes peuvent être ignorées ou non par un agent, selon qu'il est essentiellement réactif ou proactif.

La décision d'entreprendre ou non une action peut reposer sur des critères inappropriés : le sexe, l'âge, la religion, l'apparence, les convictions politiques, bref tout préjugé que pourraient entretenir des agents policiers (Kappeler et al., 1998). Dans les faits, ce qui nuit au système judiciaire, ce n'est pas tant la discrétion, que son exercice inapproprié.

D'après Gaines et ses collaborateurs (1999), si les chercheurs ne s'entendent pas sur la façon dont se développent les préjugés, la plupart les distinguent conceptuellement de la discrimination. Les préjugés sont le reflet personnel de valeurs et d'attitudes acquises à travers le processus de socialisation. Nous portons des jugements sur l'individu ou ses comportements en fonction de ce que nous croyons normal ou acceptable. Les préjugés sont souvent perçus de manière négative au moment où la personne qui les entretient se déclare pour ou contre quelque chose, alors que la discrimination se rencontre lorsqu'un agent agit ouvertement sur la base de préjugés, et lorsque son intervention a des conséquences défavorables pour la personne qui en est victime.

L'ensemble des études empiriques établissent que les variables déterminantes de la décision d'intervenir dans une situation donnée sont la gravité de l'acte commis et les antécédents de son auteur. Pour ce qui est des délits relativement moins graves (vol simple, voies de fait simples, etc.) commis par un délinquant primaire ou un délinquant connu de sa victime, la discrétion est plus grande. Les caractéristiques du décideur, la situation et les mesures extrajudiciaires jouent un rôle important dans la décision (Gottfredson et Gottfredson, 1988). 
Selon d'autres études, les plaintes sont prises plus au sérieux lorsqu'elles sont portées par des adultes (Dunham et Alpert, 1989 ; Gaines et al., 1999).

Une étude québécoise récente révèle que les agents de police des grandes agglomérations urbaines font un usage plus strict de leur pouvoir de mise en accusation dans les quartiers à plus forte composante multiculturelle (Tremblay et al., 1999).

Le statut socioéconomique, le sexe et l'état de santé mentale du délinquant et de la victime influent également sur la manière de répondre à la demande de service et la décision d'entreprendre des actions (Finn et Stalans, 1997).

Par ailleurs, les agents obéissent à deux ensembles de normes : les normes formelles enseignées dans les écoles de police et les normes informelles véhiculées par les pairs. Ces dernières peuvent influencer subtilement l'agent ou exercer sur lui une pression de conformité, en lui faisant adopter un certain comportement (Westley, 1970 ; Lundman, 1979 ; Ericson, 1982). L'approbation ou la désapprobation des pairs lui indique si l'action est acceptable ou non (Kappeler et al., 1998). De plus, le message véhiculé par les médias, particulièrement en matière de crimes, a un impact significatif sur l'opinion publique, les politiques et le système judiciaire (Chermak, 1995).

\section{Les programmes de mesures de rechange}

Au Québec, les programmes de mesures de rechange sont des programmes officiels applicables aux adolescents auxquels une infraction au Code criminel est reprochée. Autorisés par le ministère de la Justice et le ministère de la Santé et des Services sociaux, en vertu de l'article 4 de la Loi sur les jeunes contrevenants, ces programmes confient à un directeur provincial ou à toute personne désignée par ce dernier la responsabilité de préconiser des mesures de rechange, et accordent à l'agent de police le pouvoir de recommander ces mesures de substitution au terme de son enquête. Ces programmes peuvent être mis en œuvre avant le dépôt de la dénonciation, encore que le substitut du procureur général soit autorisé à procéder à un renvoi après le dépôt, dans les cas où le directeur provincial n'a pas encore évalué l'admissibilité 6 .

6. Article $12 \mathrm{du}$ Programme de mesures de rechange (Québec). 
La présentation des résultats des études de la discrétion a pour but de susciter un questionnement sur l'accroissement rapide de ce qui est d'emblée attribué au processus dit de " déjudiciarisation ", processus qui prête d'ailleurs à confusion dans les études statistiques ${ }^{7}$. On constate que ce vocable semble s'appliquer aussi bien aux cas ne donnant lieu à aucune action de la part de la police, qu'à ceux auxquels sont appliquées des mesures programmées. Par souci de rigueur, de justice et d'efficacité de la sanction, ne devrait-on pas réserver l'appellation aux cas où on applique une mesure prévue à un programme reconnu qui se veut une solution de rechange au traitement du délinquant à l'intérieur du système judiciaire traditionnel et parler de « non-judiciarisation » dans les cas de classements de dossier sans suite?

\section{Conclusion}

Nous observons effectivement une diminution du nombre total d'auteurs présumés d'infractions au Code criminel chez les jeunes Québécois. Mais c'est chez les auteurs présumés de crimes contre la propriété que le recul est surtout évident. Les auteurs présumés de crimes de violence sont, eux, en augmentation sensible. Ce qui frappe surtout, c'est l'évolution du traitement des jeunes. En 1998, année de l'enregistrement d'une diminution des jeunes accusés de $38 \%$ par rapport à 1991 , le nombre de jeunes traités hors cour a grimpé à $87 \%$. Cette tendance, qui s'est amorcée au début des années 80 , s'est encore accentuée dans les années 90 , époque par excellence de l'équilibre budgétaire. Il est vrai que l'approche favorisant l'utilisation de mesures moins coercitives que celles de nature judiciaire et l'intégration moins stigmatisante des jeunes à la communauté jouit, au Québec, de solides appuis chez nombre $d$ 'intervenants auprès des jeunes (Coalition pour la justices des mineurs, 1999). Toutefois, le traitement hors cour des jeunes s'accroît à une telle allure, que nous sommes forcés de nous interroger

7. Par exemple dans le Juristat de Statistique Canada, Mesures de rechange pour les jeunes au Canada (1999), on peut lire à la page 2 : "La déjudiciarisation peut prendre deux formes : exercice du pouvoir discrétionnaire de la police ou application de mesures de rechange $[\ldots]$ "; à la page 6 on lit : " $[\ldots]$ il y a trois voies possibles pour un jeune soupçonné d'une infraction criminelle : à la discrétion de la police, le jeune peut faire l'objet d'une déjudiciarisation, il peut être tenvoyé à des mesures de rechange ou être traduit devant un tribunal $\%$. 
sur l'évolution des critères de déjudiciarisation/non-judiciarisation des cas.

Si le facteur premier du renvoi devant la justice de l'auteur d'un acte criminel est la gravité objective de cet acte, d'autres variables (sexe, âge, race, situation socioéconomique, religion, etc.) peuvent influer sur la décision du poursuivant et mener à des abus, c'est-à-dire à des injustices.

Une analyse plus détaillée des trois grandes catégories d'infractions pourrait mettre en évidence, à l'intérieur de chacune, les types de crimes particulièrement susceptibles d'entraîner un traitement hors cour.

Une étude, d'une part, des fichiers de jeunes auteurs présumés constitués, ces dernières années, par les services de police et, d'autre part, des facteurs qui, pour une même infraction, entraînent tantôt une accusation tantôt un traitement hors cour, pourrait nous éclairer sur la nature du pouvoir discrétionnaire exercé, son encadrement et sa pertinence. Nous savons déjà par Tremblay et al. (1999) que, dans certains quartiers à forte composante multiculturelle, les policiers appliquent plus strictement la loi ; par Finn et Stalans (1997) et Kappeler et al. (1998), que le statut socioéconomique et divers autres paramètres (âge, race, religion, etc.) influent également sur leurs décisions, sans compter les normes informelles véhiculées par les pairs. L'étude exhaustive des motivations des sanctions reste par conséquent à faire.

\section{Références}

Doob, A.N., \& Chan, J.B.L. (1982). Factors affecting police decision to take juveniles to court. Revue canadienne de criminologie, 24, 25-37.

Carrington, P.J. (1999). Trends in youth crime in Canada. Revue canadienne de criminologie, 41 (1), 1 - 32.

Centre canadien de la statistique juridique. (1999). Statistique de la criminalité au Canada 1998. Ottawa : Centre canadien de la statistique juridique, Statistique Canada.

Chermak, S.M. (1995). Victims in the news: Crime and the American news media. Boulder, $\mathrm{CO}$ : Westview Press.

Coalition pour la justice des mineurs. (1999). Un système de justice pénale pour les adolescents ou contre les adolescents? Commentaire sur le projet de Loi sur le système de justice pénale pour les adolescents.

Davis, K.C. (1969). Discretionary justice : A preliminary inquiry Westport, CT : Greenwood Press.

Dunham, R.G., \& Alpert, G.P. (1989). Critical issues in policing : Contemporary readings. Prospect Heights, IL : Waveland Press. 
Ericson, R. (1982). Reproducing order : A study of police patrol work. Toronto, $\mathrm{CN}$ : University of Toronto Press.

Finn, M.A., \& Stalans, L.J. (1997). Influence of gender and mental state on police decisions in domestic assault cases. Criminal Justice and Behavior, 24 (2), 157-176.

Gaines, L.K., Kappeler V.E., \& Vaughn, J.B. (1999). Policing in America. Anderson publishing co.

Gottfredson, M.R., \& Gottfredson, D.M. (1988). Decision making in the criminal justice system: Toward the rational exercise of discretion. Law, society and policy Vol. 3. New-York : Prenum.

Kappeler, V.E., Sluder, R., \& Alpert, G.P. (1998). Forces of deviance: Understanding the dark side of policing, Second Edition. Prospect Heights, Il : Waveland Press.

Le Blanc, M., \& Beaumont, H. (1988). The Quebec perspective on the Young Offenders Act : Implementation before adoption. In J. Hudson, J.P. Hornick \& B.A. Burrows (eds), Justice and the young offender in Canada. Toronto : Wall and Thompson.

Le Blanc, M., \& Beaumont, H. (1992). The effectiveness of juvenile justice in Québec : A natural experiment in implementing formal diversion and a justice model. In R.R. Corrado, N. Bala, R. Linden \& M. Le Blanc (eds), Juvenile Justice in Canada. Toronto : Butterworths.

Lundman, R.J. (1979). Organizational norms and police discretion : An observational study of police wotk with traffic violators. Criminology, 17, 159-171.

MacKillop, B. (1998). Les mesures de rechange au Canada. Programme des Services Correctionnels, Centre Canadien de la Statistique Juridique.

Ministère de la Sécurité Publique. (1999). Statistiques 1998, Code Criminel, Autres Lois Fédérales, Lois Provinciales (sauf circulation).

Reiss, A. (1971). The police and the public. New Haven, CT : Yale University Press.

Savoie, J. (1999). La criminalité de violence chez les jeunes. Juristat. Centre canadien de la statistique juridique. Statistique Canada - No 85-002-XPF. Vol. 19 no 13 .

Statistique Canada. (1988). Manuel de la déclaration uniforme de la criminalité. Centre canadien de la statistique juridique.

Statistique Canada. (1998). Statistique de la criminalité au Canada, 1998. Centre canadien de la statistique juridique. Statistique Canada — No 85-205-XPF.

Tremblay, P., Tremblay, M., \& Léonard L. (1999). Arrestation, discrimination raciale et relations inter-groupes. Revue canadienne de criminologie, 41 (4), $457-478$.

Westley, W.A. (1970). Violence and the police: A sociological study of law, custom and morality. Cambridge, MA : MIT Press. 
TABLEAU 1

Auteurs présumés de 12 à 17 ans accusés et traités hors cour

\begin{tabular}{|c|c|c|c|c|c|c|c|c|c|}
\hline & \multicolumn{9}{|c|}{1998} \\
\hline & \multirow[b]{2}{*}{ Accusés } & \multicolumn{2}{|c|}{ Variation } & \multirow{2}{*}{$\begin{array}{l}\text { Hors } \\
\text { cour }\end{array}$} & \multicolumn{2}{|c|}{ Variation } & \multirow[b]{2}{*}{ Total } & \multicolumn{2}{|c|}{ Variation } \\
\hline & & $98 / 97$ & $98 / 91$ & & $98 / 97$ & $98 / 91$ & & $98 / 97$ & $98 / 91$ \\
\hline Violence & 2979 & 5,71 & 9,52 & 2837 & 10,05 & 149,08 & 5816 & 7,78 & 50,71 \\
\hline Propriété & 6446 & $-12,70$ & $-52,32$ & 7772 & $-10,33$ & 106,65 & 14218 & $-11,42$ & $-17,72$ \\
\hline Autres crimes & 3271 & $-6,59$ & $-26,59$ & 4191 & $-7,83$ & 33,81 & 7463 & $-7,29$ & $-1,66$ \\
\hline Total - Code criminel & 12697 & $-7,35$ & $-38,65$ & 14800 & $-6,28$ & 84,26 & 27497 & $-6,78$ & $-4,28$ \\
\hline
\end{tabular}

\begin{tabular}{|c|c|c|c|c|c|c|c|c|c|}
\hline & \multicolumn{9}{|c|}{1997} \\
\hline & \multirow[b]{2}{*}{ Accusés } & \multicolumn{2}{|c|}{ Variation } & \multirow{2}{*}{$\begin{array}{l}\text { Hors } \\
\text { cour }\end{array}$} & \multicolumn{2}{|c|}{ Variation } & \multirow[b]{2}{*}{ Total } & \multicolumn{2}{|c|}{ Variation } \\
\hline & & $97 / 96$ & $97 / 91$ & & $97 / 96$ & $97 / 91$ & & $97 / 96$ & $97 / 91$ \\
\hline Violence & 2818 & $-2,99$ & 3,60 & 2578 & 6,97 & 126,34 & 5396 & 1,52 & 39,83 \\
\hline Propriété & 7384 & $-19,59$ & $-45,38$ & 8667 & $-1,14$ & 130,44 & 16051 & $-10,58$ & $-7,11$ \\
\hline Autres crimes & 3503 & $-7,91$ & $-21,40$ & 4547 & 5,13 & 45,18 & 8050 & $-0,97$ & 6,07 \\
\hline Total - Code criminel & 13705 & $-13,76$ & $-33,78$ & 15792 & 1,87 & 96,61 & 29497 & $-6,04$ & 2,68 \\
\hline
\end{tabular}


TABLEAU 2

Auteurs présumés de 12 à 17 ans accusés et traités hors cour - taux par 100000 jeunes de 12 à 17 ans

\begin{tabular}{|c|c|c|c|c|c|c|c|c|c|}
\hline & \multicolumn{9}{|c|}{1998} \\
\hline & \multirow[b]{2}{*}{ Accusés } & \multicolumn{2}{|c|}{ Variation } & \multirow{2}{*}{$\begin{array}{l}\text { Hors } \\
\text { cour }\end{array}$} & \multicolumn{2}{|c|}{ Variation } & \multirow[b]{2}{*}{ Total } & \multicolumn{2}{|c|}{ Variation } \\
\hline & & $98 / 97$ & $98 / 91$ & & $98 / 97$ & $98 / 91$ & & $98 / 97$ & $98 / 91$ \\
\hline Violence & 529,22 & 8,00 & 11,27 & 504,00 & 12,43 & 153,06 & 1033,22 & 10,12 & 53,12 \\
\hline Propriēté & 1145,14 & $-10,81$ & $-51,55$ & 1380,71 & $-8,38$ & 109,95 & 2525,85 & $-9,50$ & $-16,40$ \\
\hline Autres crimes & 581,28 & $-4,57$ & $-25,41$ & 744,54 & $-5,83$ & 35,95 & 1325,81 & $-5,28$ & $-0,09$ \\
\hline Total - Code criminel & 2255,64 & $-5,35$ & $-37,67$ & 2629,24 & $-4,25$ & 87,21 & 4884,88 & $-4,76$ & $-2,75$ \\
\hline
\end{tabular}

\begin{tabular}{|c|c|c|c|c|c|c|c|c|c|}
\hline & \multicolumn{9}{|c|}{1997} \\
\hline & \multirow[b]{2}{*}{ Accusés } & \multicolumn{2}{|c|}{ Variation } & \multirow{2}{*}{$\begin{array}{l}\text { Hors } \\
\text { cour }\end{array}$} & \multicolumn{2}{|c|}{ Variation } & \multirow[b]{2}{*}{ Total } & \multicolumn{2}{|c|}{ Variation } \\
\hline & & $97 / 96$ & $97 / 91$ & & $97 / 96$ & $97 / 91$ & & $97 / 96$ & $97 / 91$ \\
\hline Violence & 490,00 & $-1,58$ & 3,03 & 448,27 & 8,53 & 125,08 & 938,27 & 3,01 & 39,05 \\
\hline Propriété & 1283,95 & $-18,42$ & $-45,68$ & 1507,04 & 0,30 & 129,16 & 2790,99 & $-9,27$ & $-7,62$ \\
\hline Autres crimes & 609,11 & $-6,57$ & $-21,84$ & 790,65 & 6,67 & 44,37 & 1399,76 & 0,47 & 5,48 \\
\hline Total - Code criminel & 2383,06 & $-12,50$ & $-34,14$ & 2745,96 & 3,36 & 95,52 & 5129,02 & $-4,67$ & 2,11 \\
\hline
\end{tabular}


TABLEAU 3

Auteurs présumés de 12 à 17 ans accusés et traités hors cour

\begin{tabular}{|c|c|c|c|c|c|c|c|c|c|}
\hline & \multicolumn{9}{|c|}{1996} \\
\hline & \multirow[b]{2}{*}{ Accusés } & \multicolumn{2}{|c|}{ Variation } & \multirow{2}{*}{$\begin{array}{l}\text { Hors } \\
\text { cour }\end{array}$} & \multicolumn{2}{|c|}{ Variation } & \multirow[b]{2}{*}{ Total } & \multicolumn{2}{|c|}{ Variation } \\
\hline & & $96 / 95$ & $96 / 91$ & & $96 / 95$ & $96 / 91$ & & $96 / 95$ & $96 / 91$ \\
\hline Violence & 2905 & $-4,03$ & 6,80 & 2410 & 6,35 & 111,59 & 5315 & 0,42 & 37,73 \\
\hline Propriété & 9183 & 6,16 & $-32,07$ & 8767 & 13,37 & 133,10 & 17950 & 9,56 & 3,88 \\
\hline Autres crimes & 3804 & 0,24 & $-14,65$ & 4325 & 7,40 & 38,09 & 8129 & 3,92 & 7,12 \\
\hline Total - Code criminel & 15892 & 2,71 & $-23,21$ & 15502 & 10,52 & 93,00 & 31394 & 6,43 & 9,28 \\
\hline
\end{tabular}

\begin{tabular}{|c|c|c|c|c|c|c|c|c|c|}
\hline & \multicolumn{9}{|c|}{1995} \\
\hline & \multirow[b]{2}{*}{ Accusés } & \multicolumn{2}{|c|}{ Variation } & \multirow{2}{*}{$\begin{array}{l}\text { Hors } \\
\text { cour }\end{array}$} & \multicolumn{2}{|c|}{ Variation } & \multirow[b]{2}{*}{ Total } & \multicolumn{2}{|c|}{ Variation } \\
\hline & & $95 / 94$ & $95 / 91$ & & $95 / 94$ & $95 / 91$ & & $95 / 94$ & $95 / 91$ \\
\hline Violence & 3027 & 1,82 & 11,29 & 2266 & 3,94 & 98,95 & 5293 & 2,72 & 37,16 \\
\hline Propriété & 8650 & $-1,03$ & $-36,01$ & 7733 & 19,04 & 105,61 & 16383 & 7,53 & $-5,19$ \\
\hline Autres crimes & 3795 & 10,03 & $-14,85$ & 4027 & 12,36 & 28,58 & 7822 & 11,22 & 3,07 \\
\hline Total - Code criminel & 15472 & 2,04 & 25,24 & 14026 & 14,40 & 74,63 & 29498 & 7,57 & 2,68 \\
\hline
\end{tabular}


TAB LEA U 4

Auteurs présumés de 12 à 17 ans accusés et traités hors cour — taux par 100000 jeunes de 12 à 17 ans

\begin{tabular}{|c|c|c|c|c|c|c|c|c|c|}
\hline & \multicolumn{9}{|c|}{1996} \\
\hline & \multirow[b]{2}{*}{ Accusés } & \multicolumn{2}{|c|}{ Variation } & \multirow{2}{*}{$\begin{array}{l}\text { Hors } \\
\text { cour }\end{array}$} & \multicolumn{2}{|c|}{ Variation } & \multirow[b]{2}{*}{ Total } & \multicolumn{2}{|c|}{ Variation } \\
\hline & & $96 / 95$ & $96 / 91$ & & $96 / 95$ & $96 / 91$ & & $96 / 95$ & $96 / 91$ \\
\hline Violence & 497,86 & $-3,26$ & 4,68 & 413,02 & 7,21 & 107,38 & 910,88 & 1,22 & 34,99 \\
\hline Propriétė & 1573,78 & 7,02 & $-33,42$ & 1502,49 & 14,28 & 128,47 & 3076,26 & 10,45 & 1,82 \\
\hline Autres crimes & 651,93 & 1,04 & $-16,35$ & 741,22 & 8,27 & 35,35 & 1393,14 & 4,76 & 4,99 \\
\hline Total - Code criminel & 2723,56 & 3,54 & $-24,74$ & 2656,73 & 11,41 & 89,17 & 5380,29 & 7,28 & 7,11 \\
\hline
\end{tabular}

\begin{tabular}{|c|c|c|c|c|c|c|c|c|c|}
\hline & \multicolumn{9}{|c|}{1995} \\
\hline & \multirow[b]{2}{*}{ Accusès } & \multicolumn{2}{|c|}{ Variation } & \multirow{2}{*}{$\begin{array}{l}\text { Hors } \\
\text { cour }\end{array}$} & \multicolumn{2}{|c|}{ Variation } & \multirow[b]{2}{*}{ Total } & \multicolumn{2}{|c|}{ Variation } \\
\hline & & $95 / 94$ & $95 / 91$ & & $95 / 94$ & $95 / 91$ & & $95 / 94$ & $95 / 91$ \\
\hline Violence & 514,62 & 2,53 & 8,20 & 385,24 & 4,67 & 93,43 & 899,86 & 3,43 & 33,36 \\
\hline Propriété & 1470,59 & $-0,34$ & $-37,78$ & 1314,69 & 19,87 & 99,91 & 2785,28 & 8,28 & $-7,81$ \\
\hline Autres crimes & 645,19 & 10,80 & $-17,21$ & 684,63 & 13,14 & 25,01 & 1329,82 & 11,99 & 0,21 \\
\hline Total - Code criminel & 2630,40 & 2,76 & $-27,31$ & 2384,56 & 15,20 & 69,79 & 5014,96 & 8,32 & 0,16 \\
\hline
\end{tabular}




\section{TABLEAU 5}

Auteurs présumés de 12 à 17 ans accusés et traités hors cour

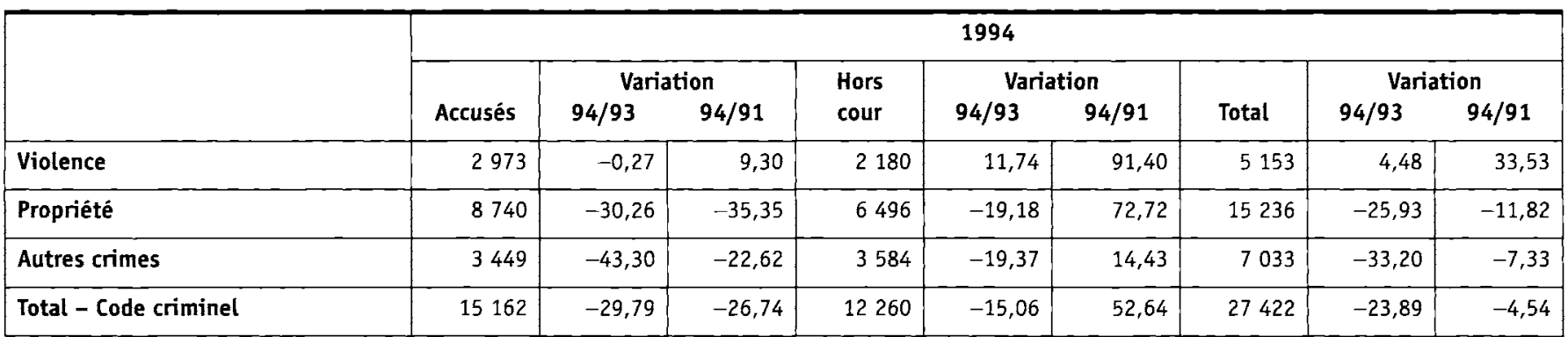

\begin{tabular}{|c|c|c|c|c|c|c|c|c|c|}
\hline & \multicolumn{9}{|c|}{1993} \\
\hline & \multirow[b]{2}{*}{ Accusés } & \multicolumn{2}{|c|}{ Variation } & \multirow{2}{*}{$\begin{array}{l}\text { Hors } \\
\text { cour }\end{array}$} & \multicolumn{2}{|c|}{ Variation } & \multirow[b]{2}{*}{ Total } & \multicolumn{2}{|c|}{ Variation } \\
\hline & & $93 / 92$ & $93 / 91$ & & $93 / 92$ & $93 / 91$ & & $93 / 92$ & $93 / 91$ \\
\hline Violence & 2981 & 1,60 & 9,60 & 1951 & 30,41 & 71,29 & 4932 & 11,33 & 27,81 \\
\hline Propriété & 12532 & 0,78 & $-7,29$ & 8038 & 75,46 & 113,72 & 20570 & 20,89 & 19,05 \\
\hline Autres crimes & 6083 & 35,27 & 36,48 & 4445 & 35,31 & 41,92 & 10528 & 35,29 & 38,73 \\
\hline Total - Code criminel & 21596 & 8,71 & 4,35 & 14434 & 54,18 & 79,71 & 36030 & 23,27 & 25,42 \\
\hline
\end{tabular}


TABLEAU 6

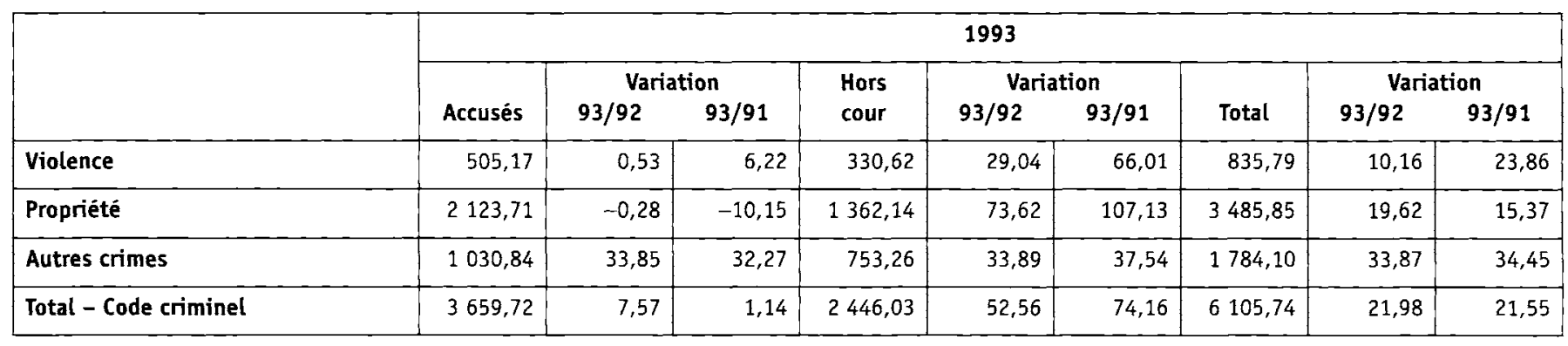


TA B LEA U 7

Auteurs présumés de 12 à 17 ans accusés et traités hors cour

\begin{tabular}{|c|c|c|c|c|c|c|c|c|c|}
\hline & \multicolumn{6}{|c|}{1992} & \multicolumn{3}{|c|}{1991} \\
\hline & Accusés & $\begin{array}{c}\text { Variation } \\
92 / 91\end{array}$ & $\begin{array}{l}\text { Hors } \\
\text { cour }\end{array}$ & $\begin{array}{c}\text { Variation } \\
92 / 91\end{array}$ & Total & $\begin{array}{c}\text { Variation } \\
92 / 91\end{array}$ & Accusés & $\begin{array}{l}\text { Hors } \\
\text { cour }\end{array}$ & Total \\
\hline Violence & 2934 & 7,87 & 1496 & 31,34 & 4430 & 14,80 & 2720 & 1139 & 3859 \\
\hline Propriété & 12435 & $-8,01$ & 4581 & 21,80 & 17016 & $-1,52$ & 13518 & 3761 & 17279 \\
\hline Autres crimes & 4497 & 0,90 & 3285 & 4,89 & 7782 & 2,54 & 4457 & 3132 & 7589 \\
\hline Total - Code criminel & 19866 & $-4,01$ & 9362 & 16,56 & 29228 & 1,74 & 20695 & 8032 & 28727 \\
\hline
\end{tabular}

TABLEAU 8

Auteurs présumés de 12 à 17 ans accusés et traités hors cour — taux par 100000 jeunes de 12 à 17 ans

\begin{tabular}{|c|c|c|c|c|c|c|c|c|c|}
\hline & \multicolumn{6}{|c|}{1992} & \multicolumn{3}{|c|}{1991} \\
\hline & Accusés & $\begin{array}{c}\text { Variation } \\
92 / 91\end{array}$ & $\begin{array}{l}\text { Hors } \\
\text { cour }\end{array}$ & $\begin{array}{c}\text { Variation } \\
92 / 91\end{array}$ & Total & $\begin{array}{c}\text { Variation } \\
92 / 91\end{array}$ & Accusés & $\begin{array}{l}\text { Hors } \\
\text { cour }\end{array}$ & Total \\
\hline Violence & 502,48 & 5,65 & 256,21 & 28,64 & 758,69 & 12,44 & 475,61 & 199,16 & 674,77 \\
\hline Propriété & 2129,65 & $-9,90$ & 784,55 & 19,30 & 2914,20 & $-3,55$ & 2363,70 & 657,63 & 3021,33 \\
\hline Autres crimes & 770,17 & $-1,18$ & 562,6 & 2,73 & 1332,76 & 0,44 & 779,33 & 547,65 & 1326,98 \\
\hline Total - Code criminel & 3402,29 & $-5,98$ & 1603,36 & 14,16 & 5005,65 & $-0,35$ & 3618,64 & 1404,44 & 5023,08 \\
\hline
\end{tabular}

